\title{
Implementación de algoritmos genéticos para el diseño, optimización y selección de vigas
}

\author{
Valentín Vázquez Castillo ${ }^{1}$, Derlis Hernández Lara ${ }^{1}$, \\ Emmanuel Alejandro Merchán Cruz ${ }^{1}$, Ricardo Gustavo Rodríguez Cañizo ${ }^{1}$, \\ Edgar Alfredo Portilla Flores ${ }^{2}$ \\ ${ }^{1}$ Instituto Politécnico Nacional, \\ Escuela Superior de Ingeniería Mecánica y Eléctrica Unidad Azcapotzalco, \\ México \\ ${ }^{2}$ Instituto Politécnico Nacional, Centro de Innovación y Desarrollo Tecnológico en Cómputo, \\ México \\ \{valentinvc29, derlis392\}@ hotmail.com, \\ \{eamerchan, rgrodriguez, aportilla\}@ipn.mx
}

\begin{abstract}
Resumen. En este trabajo se presenta la utilización de heurísticas para apoyar al diseño de sistemas mecánicos, como una propuesta de metodología de diseño robusta. Se emplearon los algoritmos genéticos en el diseño, optimización y selección de vigas utilizando la metodología de diseño paramétrico, que ayuda a variar las dimensiones (parámetros) de las secciones transversales de las vigas de estudio, con el fin de optimizarlas para soportar cargas específicas sin fracturarse. Los resultados experimentales conllevan a que este tipo de implementaciones son de gran utilidad para resolver problemas de optimización numérica en el diseño y selección de vigas para diversas aplicaciones.
\end{abstract}

Palabras clave: algoritmos genéticos, optimización, diseño paramétrico, selección de vigas.

\section{Implementation of Genetic Algorithms for the Design, Optimization, and Selection of Beams}

\begin{abstract}
In this paper, the use of heuristic techniques to support the design of mechanical systems is outlined as a proposal for a robust-design methodology. Genetic algorithms were used in the design, optimization, and beams selection for a series of applications using parametric-design methodology, which help vary the dimension or parameters of the studied beams cross sections, so that the area could be optimized applying a specific force without fracture the beam. The experimental results suggest that these types of implementations are of great help when solving numeric-optimization problems in the design of mechanical systems, as well as in the areas of beams enhancement and selection for specific applications, such as the presented case study.
\end{abstract}


Valentín Vázquez Castillo, Derlis Hernández Lara, Emmanuel Alejandro Merchán Cruz, et al.

Keywords: genetic algorithms, optimization, parametric design, beams selection.

\section{Introducción}

Uno de los principales objetivos al implementar heurísticas en problemas de optimización, es el de resolver situaciones complejas y buscar soluciones factibles dentro de un intervalo definido por las cotas de diseño establecidas. Para este estudio se realiza la optimización y selección de diferentes perfiles de vigas mediante algoritmos genéticos, en donde se obtendrá la viga y el perfil adecuados para las cargas y esfuerzos requeridos en situaciones específicas.

En Biomecánica se emplean técnicas de cómputo inteligente como lo son: algoritmos genéticos, redes neuronales y lógica difusa para la optimización en la síntesis de mecanismos para prótesis robóticas. Una tesis con este enfoque es "Diseño de mecanismos utilizando algoritmos genéticos con aplicación en prótesis para miembro inferior" donde la autora encontró las longitudes de los eslabones y la medida de los ángulos para un mecanismo óptimo con el que se reproduzca el proceso de marcha humana como caso de aplicación [1].

En el uso de aplicaciones tecnológicas como el Sistema de Posicionamiento Global (GPS por sus siglas en ingles "Global Positioning System"), la implementación de algoritmos genéticos permite optimizar el tiempo y la selección de la mejor ruta para llegar a un destino específico [2].

En trabajos como el presentado en [3] se aborda el diseño óptimo de columnas de concreto reforzado haciendo uso de un algoritmo genético, con el cual se minimiza el costo de una viga considerando no sólo los esfuerzos permisibles por el elemento, sino también los costos del concreto, el acero y el recubrimiento utilizados. El modelo de diseño óptimo se basa en el propuesto por Chakrabarty [4], aunque incluye ciertas modificaciones que lo hacen más apropiado para aplicaciones prácticas.

En [5] se han aplicado métodos de optimización con criterios múltiples a problemas de diseño de vigas. Asimismo, en [6] propusieron un modelo para optimizar el diseño de secciones de concreto reforzado en el cual consideraron los costos del acero, el concreto y el recubrimiento.

\subsection{Algoritmos genéticos}

Los algoritmos genéticos (AG) son simulaciones de la selección natural que pueden resolver problemas de optimización, es decir, son considerados como una estrategia de búsqueda que imita la teoría de la evolución de la vida en la naturaleza. Ahora bien, si un algoritmo genético es una técnica de búsqueda iterativa inspirada en los principios de selección natural, es importante aclarar que los AG no buscan modelar la evolución biológica, sino derivar estrategias de optimización. La idea de los AG es optimizar (hallar el máximo o mínimo) una función objetivo utilizando los principios de selección natural sobre los parámetros de la función [7].

El proceso de ejecución del AG se inicia creando una población de $\boldsymbol{n}$ individuos, donde cada uno contiene dentro de su cromosoma la información del problema a 
optimizar, en este caso específico, los parámetros de diseño para cada una de las secciones transversales de una viga, es decir, sus dimensiones.

Luego se establece una función objetivo que evaluará a cada uno de los individuos para determinar su aptitud. Enseguida se realiza la operación de cruce de genes del cromosoma entre dos individuos, operación realizada mediante la ruleta de selección, la cual consiste en seleccionar aleatoriamente dos individuos con base en su aptitud, lo que implica que la probabilidad de selección será mayor conforme tenga mejor aptitud, así como aquellos individuos que tengan aptitud igual a cero serán despreciados en la selección del cromosoma del individuo para compartir genes con otro individuo y crear un descendiente.

Finalmente dependiendo del problema, el diseñador determinará la probabilidad de mutación para el AG, es decir, la modificación de los genes en el cromosoma de un individuo lo cual puede o no mejorar la aptitud del mismo, finalizando de esta forma la primera generación del AG representada en la figura 1.

Sin embargo, es importante que el número de descendientes sea igual al número de individuos de la población creada al inicio del algoritmo, ya que estos descendientes pasarán a ser la población inicial para la generación 2, repitiendo el proceso de la Figura 1 hasta encontrar los valores óptimos que satisfagan el problema a optimizar.

El pseudocódigo del AG implementado se muestra en el Algoritmo1.

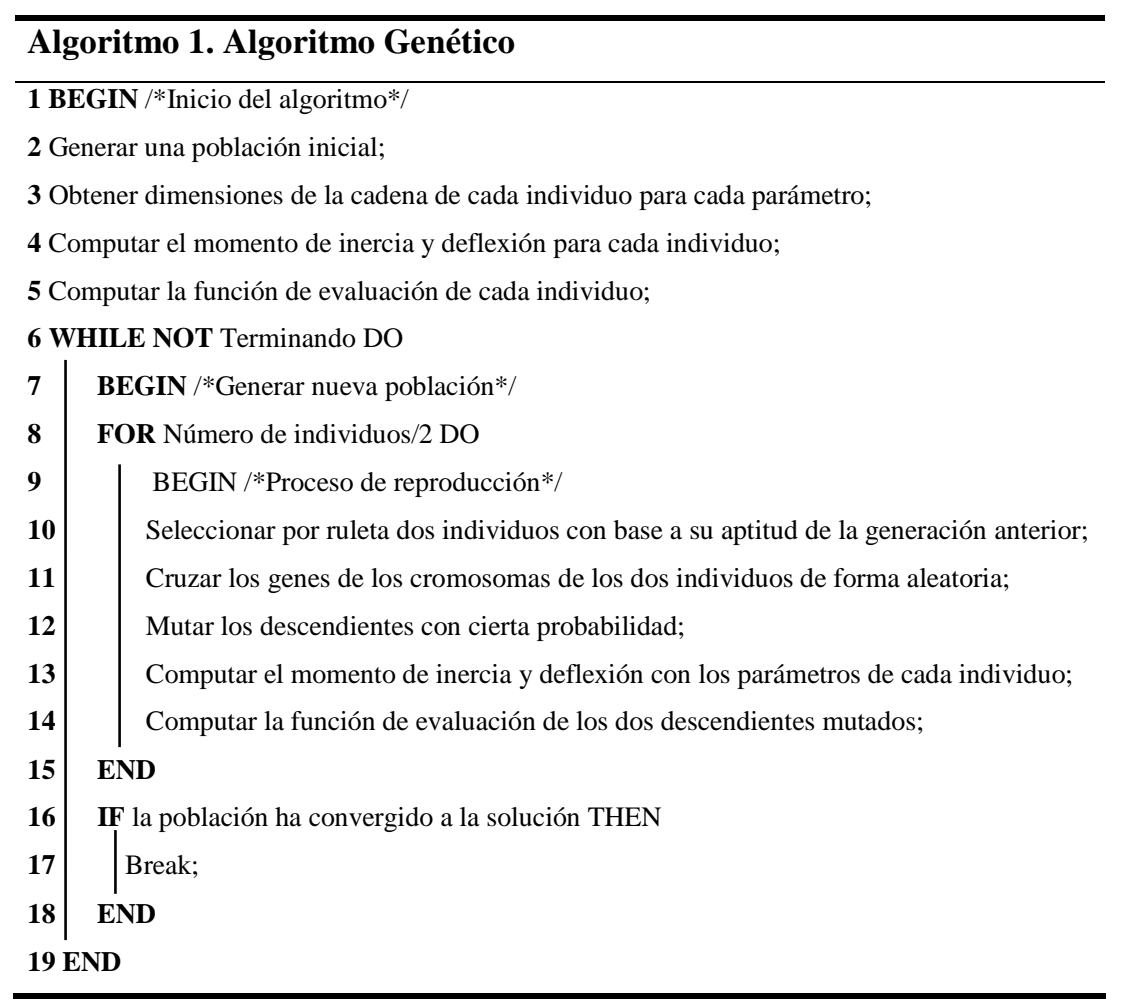




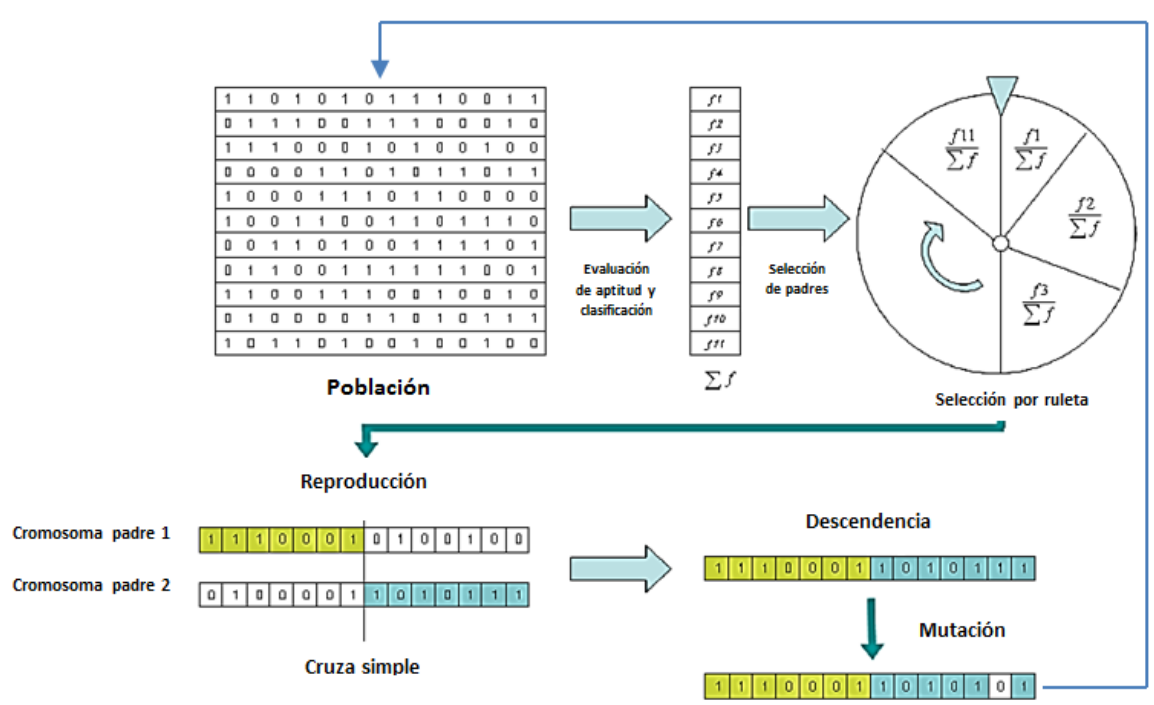

Fig. 1. Descripción general de un AG [8].

\section{Implementación computacional para la optimización de vigas}

La implementación del algoritmo se programó en el entorno de MATLAB $®$ R2015a, y las corridas se llevaron a cabo en una plataforma computacional con las siguientes características: procesador Inter Core i5 @ $1.80 \mathrm{GHz}$, con 8Gb de memoria RAM y Sistema operativo Windows 8.

Las secciones transversales que se optimizaron son tipo: rectangular (a), T (b), I (c) tubular cilíndrica (d) y tubular cuadrada (e), tal como se muestran en la figura 2.

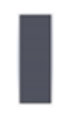

a)

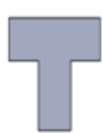

b)

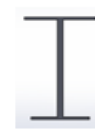

c)

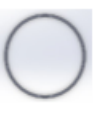

d)

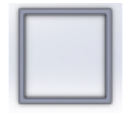

e)

Fig. 2. Secciones transversales a optimizar con el AG.

La figura 3 muestra los parámetros que definen la sección trasversal rectangular, los cuales son implementados como variables dentro del AG.

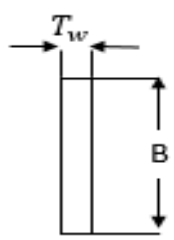

Fig. 3. Parámetros a evaluar en el AG para la sección rectangular. 
Se sabe que la deflexión máxima de vigas en voladizo se determina por la ecuación:

$$
y_{\text {máx }}=-\frac{P L^{3}}{3 E I^{\prime}}
$$

donde:

$\boldsymbol{P}$ : es la carga que se ejerce sobre la viga.

$\boldsymbol{L}:$ es la longitud de la viga.

$\boldsymbol{E}$ : es el módulo de elasticidad del material.

I: es el momento de inercia.

En el caso de la sección transversal rectangular el momento de inercia I se calcula por la ecuación:

$$
I_{x x_{S R}}=\frac{T_{w} B^{3}}{12} .
$$

Se implementó una restricción para esta sección transversal dentro del proceso del AG para obtener valores lógicos, la cual está dada por:

$$
0<\mathrm{Tw}<\mathrm{B} .
$$

De la misma forma se parametrizó cada sección trasversal con la finalidad de obtener el momento de inercia, así como el área y poder determinar la función objetivo implementada en el AG. La figura 4 muestra los parámetros que se requieren en la obtención de dichos datos del problema para cada una de las secciones.

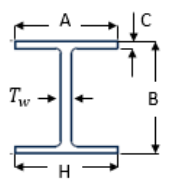

a)

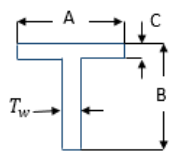

b)

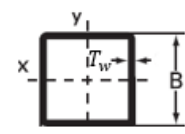

c)

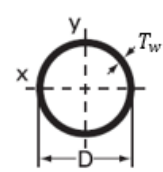

d)

Fig. 4. Parametrización de las secciones transversales de vigas a optimizar.

Para las secciones transversales tubulares circular (ecuación 4) y cuadrada (ecuación 5) se obtuvieron por fórmula y están expresadas como:

$$
\begin{gathered}
I_{x x_{S T c i}}=\frac{\pi\left(D^{2}-d^{2}\right)}{4}, \\
I_{x x_{S T c u}}=\frac{\left(S^{4}\right)}{12}-\frac{\left(s^{4}\right)}{12},
\end{gathered}
$$

Dado que las secciones transversales tipo I y $\mathbf{T}$ son perfiles compuestos, se utilizó el método del teorema del eje paralelo para el cálculo de los momentos de inercia en cada caso.

Retomando la ecuación 1 para el cálculo de deflexión de una viga en cantiléver, y considerando que se necesita determinar una deflexión máxima conocida, es decir, manejando el dato como una constante, así como también la longitud total de la viga, 
el material y la carga ejercida en el extremo de la viga tal como se muestra en la figura 5 , se despeja el momento de inercia de la ecuación y se obtiene que:

$$
\mathrm{I}=-\frac{P L^{3}}{3 E y_{m a ́ x}} \text {. }
$$

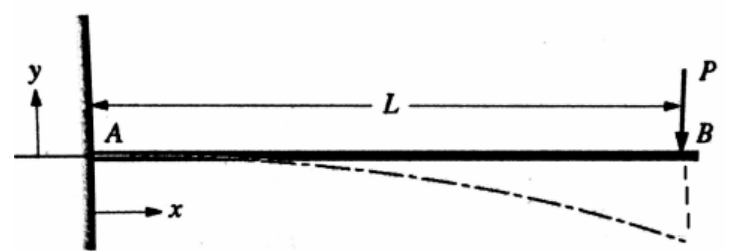

Fig. 5. Aplicación de una carga $\mathbf{P}$ en el extremo de una viga en cantiléver.

De esta forma las ecuaciones que describen los momentos de inercia, se obtienen sustituyendo los parámetros del perfil en la ecuación 4, respecto a la figura 4d para el perfil tubular cilíndrico, quedando como sigue:

$$
\begin{gathered}
I_{x x_{S T C i}}=\frac{\pi}{64}\left(D^{4}-\left(D-2 T_{w}\right)^{4}\right), \\
A_{\text {total }_{S T C i}}=\pi\left(\frac{D}{2}\right)^{2}-\left(\frac{D-2 T_{w}}{2}\right)^{2}
\end{gathered}
$$

Para el perfil tubular cuadrado, quedan las siguientes ecuaciones:

$$
\begin{aligned}
I_{x x_{S T c u}} & =\frac{\left(B^{4}\right)}{12}-\frac{\left(B-2 T_{W}\right)^{4}}{12} \\
A_{\text {total }_{S T c u}} & =B^{2}-\left(B-2 T_{W}\right)^{2}
\end{aligned}
$$

Finalmente, se plantea la generación de la población del AG por medio de los parámetros de diseño de cada sección transversal. Para esto, se crea una población inicial de 1000 individuos. En el caso del perfil rectangular, cada individuo contiene en su cadena un código binario creado aleatoriamente por 38 genes o bits, los cuales proporcionan la información de los parámetros $\boldsymbol{T}_{\boldsymbol{w}}$ y $\mathbf{B}$, donde 19 bits representan el valor para cada parámetro o cromosoma tal como se muestran en la figura 6.

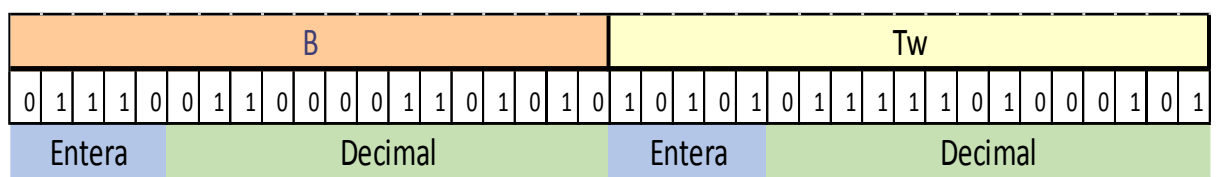

Fig. 6. Cromosomas de un individuo de la población para el cálculo de la sección transversal rectangular.

Como se observa en la figura 6 , los primeros 5 genes del cromosoma representan el valor entero y los siguientes 14 el valor decimal, proporcionando el valor total del parámetro $\mathbf{B}$. De tal forma que para tener el valor decimal de $\mathbf{B}$ se debe hacer una conversión de código binario a decimal para la parte entera y decimal, sumarlos y 
finalmente obtener su valor total para el parámetro $\mathbf{B}$ en forma de dato flotante. Estas operaciones ocurren dentro del AG para cada individuo.

Esto sucede para el resto de los perfiles, sin embargo, existen variaciones en cuanto al número de genes en los cromosomas de los individuos en cada perfil, debido a la complejidad de los parámetros de diseño que forman cada una de las distintas secciones transversales de estudio, establecidas en el Manual de Construcción en Acero, propuestas por el Instituto Mexicano de la Construcción del Acero, A.C. (IMCA) [9].

La Tabla 1 muestra el tamaño de población, así como la longitud del cromosoma que forman el resto de los perfiles estructurales para optimizar el problema de estudio.

Tabla 1. Características de la población de los diferentes perfiles de estudio.

\begin{tabular}{l|l|l}
\hline \multicolumn{1}{c|}{ Tipo de perfil } & Número de individuos & Longitud del Cromosoma \\
\hline $\mathrm{I}$ & 15,000 & 91 \\
\hline $\mathrm{T}$ & 2,500 & 72 \\
\hline Tubular circular & 1,000 & 38 \\
\hline Tubular cuadrado & 2,500 & 33 \\
\hline
\end{tabular}

Es importante tener presente que la longitud del cromosoma aumentará conforme los parámetros de diseño incrementen, y en ocasiones se necesitará de una población mayor de individuos para converger a la solución tal como se observa en la tabla 1.

Tabla 2. Restricciones para la generación de forma de los distintos perfiles.

\begin{tabular}{l|c}
\hline Perfil & Restricciones de construcción \\
\hline \multirow{4}{*}{ I } & $B<2 A y B<2 H$ \\
& $0.10 B<A<0.80 B$ \\
& $0.10 B<H<0.80 B$ \\
& $0.10<C<0.35 B$ \\
& $0.10<T_{w}<0.25 B$ \\
$\mathrm{~T}$ & $A<1.75 H \quad y \quad H<1.75 A$ \\
\hline \multirow{3}{*}{ Tubular circular } & $C \neq 0 \quad T_{w} \neq 0$ \\
& $A<B<3 A$ \\
& $B \geq 1 \quad y \geq 1$ \\
\hline \multirow{3}{*}{ Tubular cuadrado } & $D>\left(D-2 T_{w}\right)$ \\
& $\left(D-2 T_{w}\right)<0.90 D$ \\
& $D \geq 1$ \\
\hline
\end{tabular}

Tomando en consideración que la generación 1 creada aleatoriamente puede formar áreas sin lógica alguna, y con fundamento al Manual de Construcción en Acero [9] 
mencionado anteriormente, se establecieron ciertas restricciones para cada uno de los perfiles en el AG, las cuales están descritas en la tabla 2.

Finalmente, una vez obtenida la primera generación es posible que un porcentaje pequeño caiga dentro de las restricciones determinadas en la Tabla 2, sin embargo, para que las siguientes generaciones mejoren, se establece una función objetivo, la cual se encargará de evaluar la aptitud de cada individuo y en dado caso que viole alguna de las restricciones, no se tomará en consideración en la operación de cruce del AG.

La Tabla 3 muestra cada una de las funciones objetivo que se determinaron para evaluar la aptitud de los individuos de la población generada, a través del análisis de los parámetros que permiten obtener la menor área posible y logren la deflexión requerida por el problema para que no se fracture la viga, es decir, minimizando el área total a través de las dimensiones de diseño para cada perfil, así como el tamaño del error determinado por la deflexión máxima y la deflexión deseada en el problema.

De esta forma las ecuaciones de 14-18 ilustran cómo influyen los parámetros de área y el error entre la deflexión deseada, y la deflexión que se genera a través de los parámetros de diseño de viga de cada uno de los individuos generados por la población inicial y consecuente en cada generación.

Tabla 3. Función objetivo a minimizar para cada perfil estructural de vigas.

\begin{tabular}{l|l}
\hline Perfil & Función objetivo \\
\hline Rectangular & $f(x)=\frac{1}{Y_{\text {máx }}-Y Y_{\text {máx }}}+\frac{1000}{B * T_{w}}$ \\
\hline $\mathrm{I}$ & $f(x)=\frac{1 e 7}{Y_{\text {máx }}-Y Y_{\operatorname{máx}}}+\frac{1 e 9}{A+H+B+\left(A_{\text {total }}\right)}+\frac{1 e 13}{a b s\left(I_{x x}-I I_{x x}\right)}$ \\
\hline $\mathrm{T}$ & $f(x)=\frac{1 e 4}{Y_{\text {máx }}-Y Y_{\text {máx }}}+\frac{1 e 8}{A_{\text {total }}}$ \\
\hline Tubular circular & $f(x)=\frac{10}{Y_{\text {máx }}-Y Y_{\text {máx }}}+\frac{1000}{\pi\left(\frac{D}{2}\right)^{2}-\left(\frac{D-2 T_{w}}{2}\right)^{2}}$ \\
\hline Tubular cuadrada & $f(x)=\frac{10}{Y_{\text {máx }}-Y Y_{\text {máx }}}+\frac{1000}{B^{2}-\left(B-2 T_{W}\right)^{2}}$ \\
\hline
\end{tabular}

\section{Resultados}

Para analizar los resultados del algoritmo implementado, se tomaron datos de problemas ejemplo del libro Resistencia de Materiales del autor Robert L Mott [10]. Específicamente problemas de vigas en voladizo, en donde se propone usar acero estándar y soportar una deflexión máxima establecida.

El AG se implementó para tomar como base el análisis de dichos problemas y optimizar cada una de las 5 secciones transversales de estudio en este trabajo. Los datos constantes son longitud $\mathbf{L}$, carga $\mathbf{P}$, deflexión máxima $\boldsymbol{y}_{\max }$, y el módulo de elasticidad 
de un acero estándar, se obtuvieron los resultados que se muestran en la tabla 4 , los cuales cumplen con las características requeridas por el diseño.

Tabla 4. Resultados promedio del AG.

\begin{tabular}{|c|c|c|c|c|c|c|c|c|}
\hline 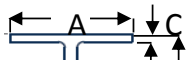 & $B($ in $)$ & $C($ in $)$ & $H($ in $)$ & $T_{w}(i n)$ & $A($ in $)$ & $A_{\text {total }}\left(i n^{2}\right)$ & $I_{x x}\left(i n^{4}\right)$ & $\gamma_{\max }($ in $)$ \\
\hline$\underset{\mathrm{H}}{T_{w} \rightarrow \leftarrow} \underset{ }{\leftarrow}$ & 4.097 & 0.1024 & 2.8392 & 0.227 & 2.3124 & 1.45754284 & 4.3214 & 0.083334 \\
\hline$\leftarrow A \rightarrow\lfloor\downarrow C$ & $T_{w}$ (in) & $B($ in $)$ & $A($ in $)$ & \multicolumn{2}{|c|}{$C($ in $)$} & $A_{\text {total }}\left(i n^{2}\right)$ & $I_{x x}\left(i n^{4}\right)$ & $\gamma_{\max }($ in $)$ \\
\hline$T_{\vec{w}} \longrightarrow$ & 1.0404 & 3.1395 & 1.0489 & \multicolumn{2}{|c|}{1.0906} & 3.2756059 & 4.3231 & 0.083335 \\
\hline & \multicolumn{3}{|c|}{$B($ in $)$} & \multicolumn{2}{|c|}{$T_{w}($ in $)$} & $A_{\text {total }}\left(\mathrm{in}^{2}\right)$ & $I_{x x}\left(i n^{4}\right)$ & $\gamma_{\max }($ in $)$ \\
\hline & \multicolumn{3}{|c|}{2.8896} & \multicolumn{2}{|c|}{0.417} & 4.1242968 & 4.322015 & 0.0832945 \\
\hline & \multicolumn{3}{|c|}{$\mathrm{D}(\mathrm{in})$} & \multicolumn{2}{|c|}{$T_{W}(\mathrm{in})$} & $A_{\text {total }}\left(\mathrm{in}^{2}\right)$ & $I_{x x}\left(i n^{4}\right)$ & $\gamma_{\max }($ in $)$ \\
\hline & \multicolumn{3}{|c|}{4.7436} & \multicolumn{2}{|c|}{0.1106} & 1.609782863 & 4.321647 & 0.0833016 \\
\hline \multirow[t]{2}{*}{$\rightarrow$} & \multicolumn{3}{|c|}{$\mathrm{B}(\mathrm{in})$} & \multicolumn{2}{|c|}{$T_{w}($ in $)$} & $A_{\text {total }}\left(\mathrm{in}^{2}\right)$ & $I_{x x}\left(i n^{4}\right)$ & $\gamma_{\max }($ in $)$ \\
\hline & \multicolumn{3}{|c|}{3.2869} & \multicolumn{2}{|c|}{1.4604} & 4.80018876 & 4.321655 & 0.0833014 \\
\hline
\end{tabular}

Tabla 5. Características del AG para converger a la solución.

\begin{tabular}{l|l|l|l}
\hline Tipo de perfil & No. de individuos & No. de generaciones & No. de corridas \\
\hline $\mathrm{I}$ & 15,000 & 17 & 50 \\
\hline $\mathrm{T}$ & 2,500 & 81 & 50 \\
\hline Tubular Circular & 1,000 & 16 & 50 \\
\hline Tubular Cuadrada & 2,500 & 16 & 50 \\
\hline Rectangular & 1,000 & 24 & 50 \\
\hline
\end{tabular}

Estos resultados son valores promedio de haber realizado múltiples corridas del algoritmo y de una selección de muestras de estudio de 50 corridas, las cuales convergieron a la solución. La tabla 5 muestra un resumen de las características del algoritmo para cada uno de los perfiles. 
Valentín Vázquez Castillo, Derlis Hernández Lara, Emmanuel Alejandro Merchán Cruz, et al.

Las figuras 7, 10, 11, 12 y 13 muestran gráficamente cómo evoluciona la solución de cada generación hasta converger a la solución óptima.

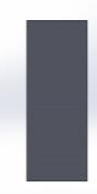

a)

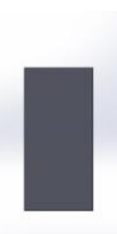

b)

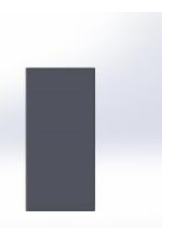

c)

Fig. 7. Comportamiento del AG para el perfil rectangular. Generación 1 (a), 6 (b) y 24 (c).

Se puede ver el salto que tienen los individuos en su valor de aptitud en la evolución del algoritmo. Este comportamiento se repite en cada una de las secciones transversales de estudio en este trabajo.

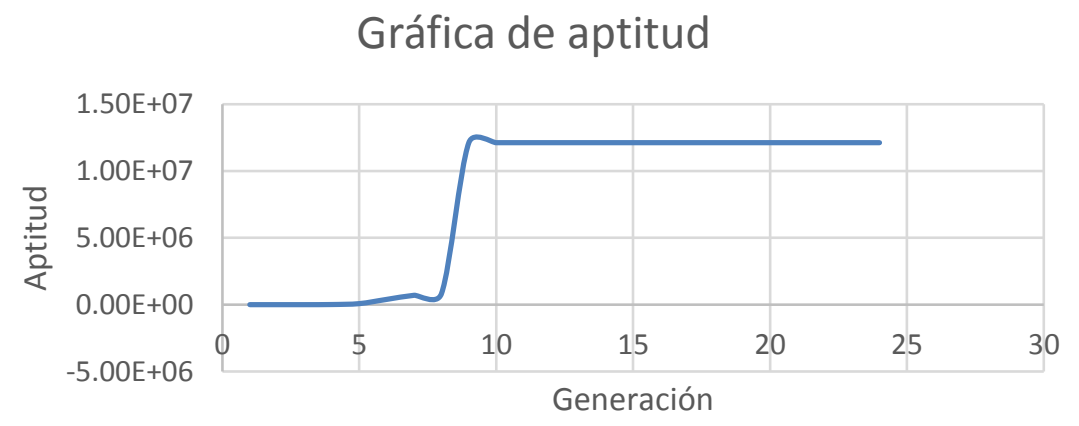

Fig. 8. Comportamiento del valor de aptitud durante la evolución del perfil rectangular en específico y en general para todos los perfiles.

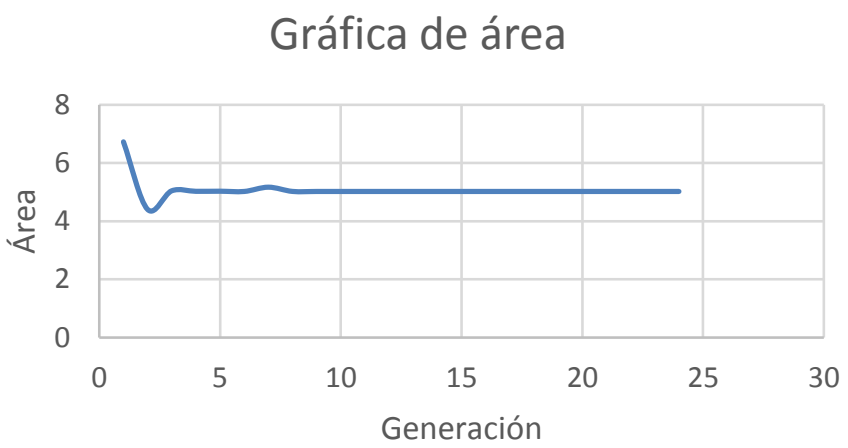

Fig. 9. Obtención del área óptima para perfil rectangular. 
Uno de los objetivos que cumple el AG implementado es optimizar la sección transversal de las vigas. Esto se obtiene conforme el algoritmo evoluciona, al encontrar el área óptima, lo cual puede observarse en las gráficas de las Figuras 9, 10, 11, 12 y 13 para cada perfil respectivamente.

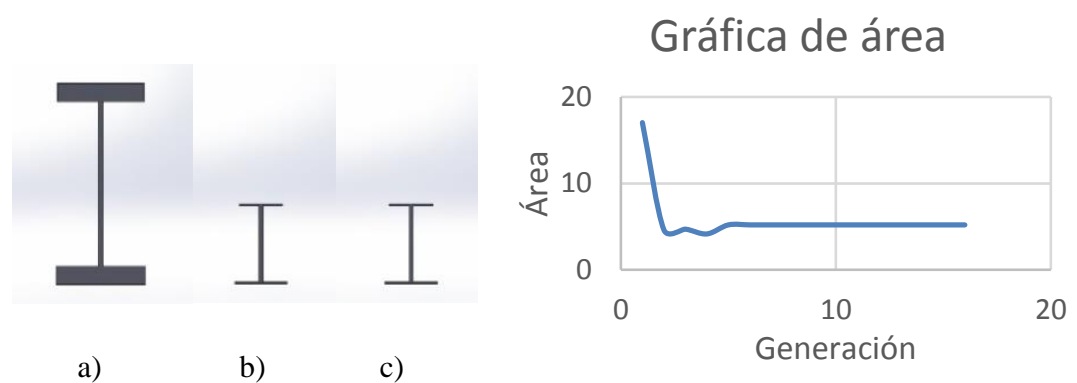

Fig. 10. Comportamiento del AG para el perfil I. Generación 1 (a), 6 (b) y 12 (c).

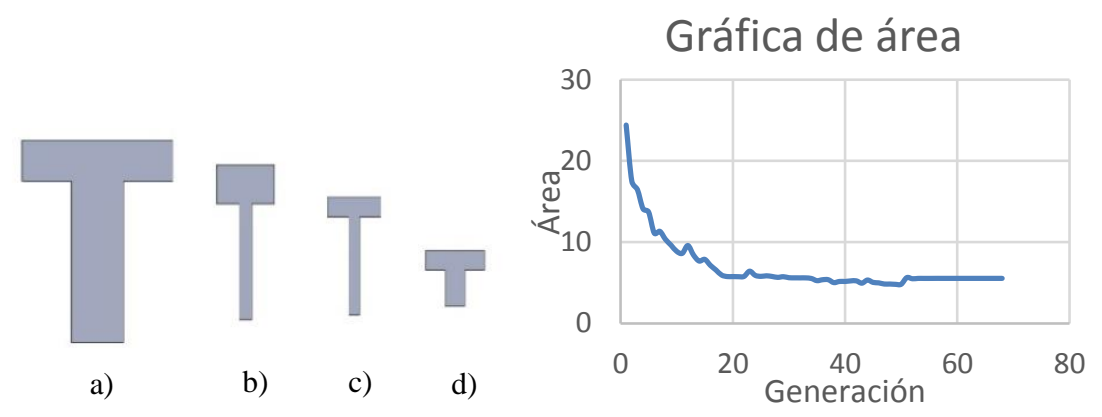

Fig. 11. Comportamiento del AG para el perfil T. Generaciones: 1(a), 13 (b), 29 (c) y 56 (d).

En los resultados de la sección transversal tipo I, mostrados geométricamente en la figura 10, se observa cómo el algoritmo minimiza los parámetros de la sección transversal entre la primera y última generación, cumpliéndose este objetivo en todas las secciones analizadas.

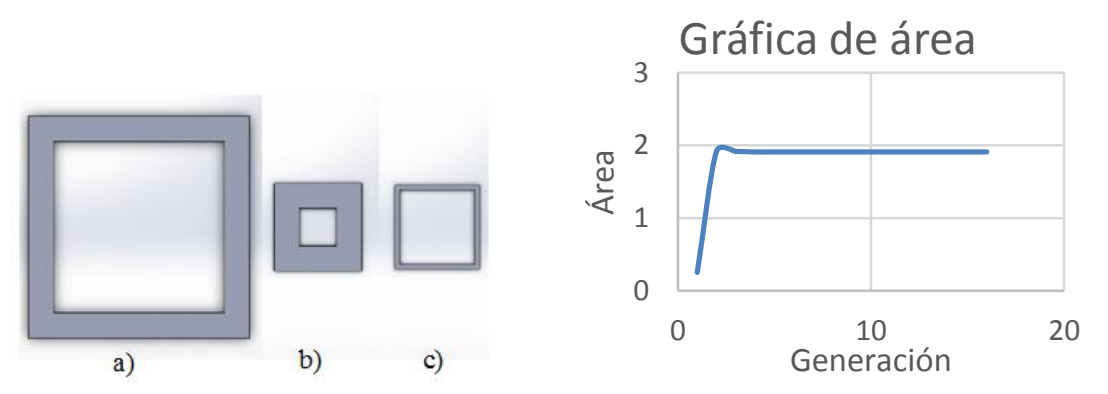

Fig. 12. Comportamiento del AG, perfil tubular cuadrado. Generaciones: 1 (a), 7 (b) y 16 (c). 


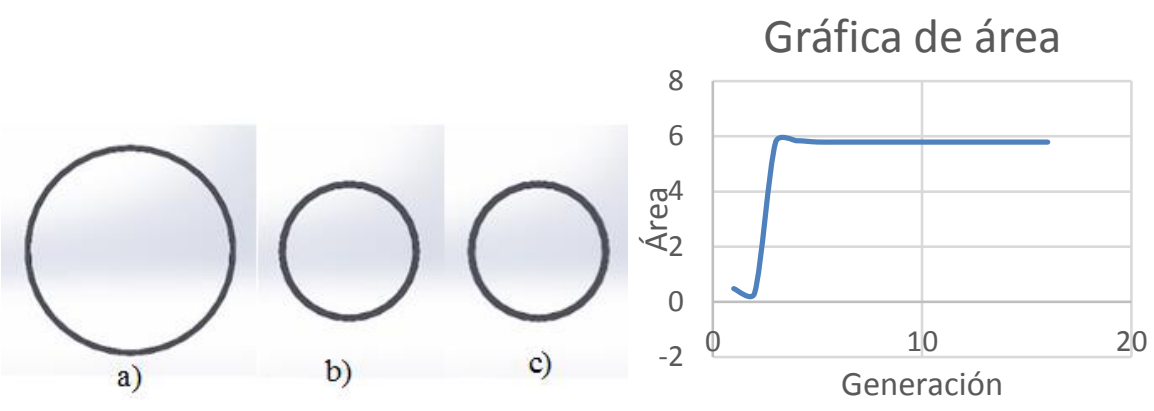

Fig. 13. Comportamiento del algoritmo, perfil tubular cilíndrico. Generaciones: 1, 7 y 16.

\subsection{Discusión}

Los resultados obtenidos por el AG implementado para cada uno de los perfiles, muestran como los individuos de cada generación mejora a la anterior, además, cumple con el objetivo de optimizar la sección transversal para cada perfil, sin embargo, es importante desarrollar cada perfil con una función objetivo diferente dentro del algoritmo genético, dado que cuando se implementó el desarrollo para las secciones transversales tipo T, I y rectangular en una sola función, los resultados del algoritmo arrojaban las características del perfil rectangular sin tener oportunidad de evaluar como usuario las características de los otros perfiles, esto para fines de diseño es de suma importancia, ya que como diseñador teniendo los valores óptimos de cada perfil, la selección de la sección transversal se elegirá de acuerdo a la aplicación a realizar.

En la tabla 6, se muestran numéricamente los valores de cada parámetro de las soluciones del algoritmo implementado, donde se puede observar las variaciones mínimas que tienen los parámetros de diseño de la sección trasversal para una viga tipo $\mathbf{T}$ de las analizadas en este trabajo para diferentes corridas del AG.

Tabla 6. Parámetros de la viga $\mathbf{T}$ dentro de 30 corridas.

\begin{tabular}{|c|c|c|c|c|c|c|c|c|c|}
\hline $\mathbf{N}$ & $\mathbf{i}$ & $\mathbf{B}$ & $\mathbf{T w}$ & $\mathbf{A}$ & $\mathbf{C}$ & $\begin{array}{c}\text { Área } \\
\text { Total }\end{array}$ & \multicolumn{1}{|c|}{$\mathbf{I}_{\mathbf{X x}}$} & $\mathbf{y}_{\max }$ & F.o. \\
\hline 1 & 68 & 3.1416 & 1.0377 & 3.1351 & 1.0929 & 5.5522 & 4.32168993 & 0.08330075 & $1.34 \mathrm{E}+10$ \\
\hline 2 & 63 & 3.4927 & 0.6552 & 2.9842 & 1.3282 & 5.3817 & 4.32173629 & 0.08329985 & $6.83 \mathrm{E}+10$ \\
\hline 3 & 54 & 3.1238 & 1.0769 & 3.0813 & 1.1216 & 5.6121 & 4.32175775 & 0.08329944 & $1.79 \mathrm{E}+10$ \\
\hline 4 & 86 & 3.3885 & 0.7496 & 2.997 & 1.2648 & 5.3825 & 4.32173934 & 0.08329979 & $4.88 \mathrm{E}+10$ \\
\hline 5 & 119 & 3.1738 & 1.0068 & 3.0386 & 1.1579 & 5.5480 & 4.32173774 & 0.08329983 & $5.74 \mathrm{E}+10$ \\
\hline 6 & 46 & 3.1492 & 1.0235 & 3.1483 & 1.0868 & 5.5324 & 4.32175008 & 0.08329959 & $2.43 \mathrm{E}+10$ \\
\hline 7 & 52 & 3.5167 & 0.6283 & 3.0641 & 1.2988 & 5.3731 & 4.32172575 & 0.08330006 & $1.76 \mathrm{E}+11$ \\
\hline 8 & 44 & 3.2493 & 0.9047 & 3.0213 & 1.191 & 5.4605 & 4.3217377 & 0.08329983 & $5.76 \mathrm{E}+10$ \\
\hline 9 & 105 & 3.442 & 0.6879 & 3.0979 & 1.237 & 5.3489 & 4.32181104 & 0.08329841 & $6.32 \mathrm{E}+09$ \\
\hline 10 & 221 & 3.753 & 0.5238 & 2.5988 & 0.7155 & 3.4504 & 4.32175447 & 0.0832995 & $2.02 \mathrm{E}+10$ \\
\hline
\end{tabular}


Implementación de algoritmos genéticos para el diseño, optimización y selección de vigas

\begin{tabular}{|c|c|c|c|c|c|c|c|c|c|}
\hline 11 & 32 & 3.3553 & 0.7556 & 3.2292 & 1.1185 & 5.3019 & 4.32174541 & 0.08329968 & $3.10 \mathrm{E}+10$ \\
\hline 12 & 70 & 3.4561 & 0.6994 & 2.8696 & 1.371 & 5.3925 & 4.32169511 & 0.08330065 & $1.55 \mathrm{E}+10$ \\
\hline 13 & 32 & 3.4196 & 0.6976 & 3.2002 & 1.1683 & 5.3093 & 4.3217289 & 0.0833 & $2.54 \mathrm{E}+12$ \\
\hline 14 & 138 & 3.5381 & 0.6283 & 2.8707 & 1.4169 & 5.4002 & 4.32175733 & 0.08329945 & $1.81 \mathrm{E}+10$ \\
\hline 15 & 59 & 3.5362 & 0.6197 & 2.9849 & 1.3536 & 5.3929 & 4.32170043 & 0.08330054 & $1.84 \mathrm{E}+10$ \\
\hline 16 & 166 & 3.3741 & 0.7851 & 2.8391 & 1.3471 & 5.4159 & 4.32170348 & 0.08330049 & $2.06 \mathrm{E}+10$ \\
\hline 17 & 78 & 3.4829 & 0.6685 & 2.9341 & 1.3501 & 5.3871 & 4.32168552 & 0.08330083 & $1.20 \mathrm{E}+10$ \\
\hline 18 & 60 & 3.136 & 1.0496 & 3.1192 & 1.1012 & 5.5705 & 4.32177321 & 0.08329914 & $1.17 \mathrm{E}+10$ \\
\hline 19 & 1 & 3.284 & 0.8466 & 3.122 & 1.1463 & 5.3885 & 4.3217148 & 0.08330027 & $3.74 \mathrm{E}+10$ \\
\hline 20 & 31 & 3.3185 & 0.8015 & 3.1671 & 1.1357 & 5.3463 & 4.32170985 & 0.08330036 & $2.76 \mathrm{E}+10$ \\
\hline 21 & 32 & 3.2794 & 0.8401 & 3.2083 & 1.0953 & 5.3489 & 4.32168293 & 0.08330088 & $1.14 \mathrm{E}+10$ \\
\hline 22 & 64 & 3.1651 & 1.0092 & 3.0952 & 1.1221 & 5.5349 & 4.32173765 & 0.08329983 & $5.80 \mathrm{E}+10$ \\
\hline 23 & 61 & 3.1181 & 1.0791 & 3.1165 & 1.0994 & 5.6046 & 4.32175945 & 0.08329941 & $1.69 \mathrm{E}+10$ \\
\hline 24 & 69 & 3.1595 & 1.0206 & 3.0798 & 1.1299 & 5.5512 & 4.32178357 & 0.08329894 & $9.47 \mathrm{E}+09$ \\
\hline 25 & 46 & 3.1528 & 1.0181 & 3.1479 & 1.0879 & 5.5268 & 4.32180765 & 0.08329848 & $6.59 \mathrm{E}+09$ \\
\hline 26 & 460 & 3.3959 & 0.7684 & 2.7922 & 1.384 & 5.4103 & 4.32177755 & 0.08329906 & $1.06 \mathrm{E}+10$ \\
\hline 27 & 91 & 3.4161 & 0.7255 & 2.9717 & 1.293 & 5.3827 & 4.32173978 & 0.08329979 & $4.68 \mathrm{E}+10$ \\
\hline 28 & 78 & 3.1531 & 1.0261 & 3.1023 & 1.115 & 5.5503 & 4.32170436 & 0.08330047 & $2.13 \mathrm{E}+10$ \\
\hline 29 & 63 & 3.387 & 0.7358 & 3.1276 & 1.1911 & 5.3410 & 4.32172872 & 0.0833 & $1.55 \mathrm{E}+13$ \\
\hline 30 & 39 & 3.4507 & 0.7098 & 2.823 & 1.3941 & 5.3953 & 4.32169265 & 0.08330069 & $1.44 \mathrm{E}+10$ \\
\hline
\end{tabular}

\section{Conclusiones}

Se ha logrado implementar un algoritmo que es capaz de optimizar cinco secciones transversales para el análisis de una viga en cantiléver sometida a una carga en el extremo, de esta manera, el diseñador podrá ahorrar tiempo en el proceso de diseño cuando requiera de un análisis de este tipo de elementos, variando dentro del código del algoritmo la longitud $\mathbf{L}$ de la viga, así como la carga $\mathbf{P}$ sometida en el extremo.

\section{Trabajo a futuro}

Con el fin de continuar con el desarrollo alcanzado por este trabajo, se propone implementar otros perfiles estructurales, con el objetivo de tener un catálogo final de las dimensiones de cada sección transversal y así el diseñador pueda hacer uso de los resultados con base a la aplicación específica.

Agradecimientos. Los autores agradecen al Instituto Politécnico Nacional, a la Sección de Estudios de Posgrado e Investigación de la Escuela Superior de Ingeniería Mecánica 
Valentín Vázquez Castillo, Derlis Hernández Lara, Emmanuel Alejandro Merchán Cruz, et al.

y Eléctrica Unidad Azcapotzalco, y al Consejo Nacional de Ciencia y Tecnología por el apoyo brindado.

\section{Referencias}

1. González, E. L.: Diseño de Mecanismos utilizando algoritmos geneticos con aplicación en protesis para miembro inferior. Instituto Politécnico Nacional, México, 1-204 (2010)

2. Abed, M. M., Mohd, G. A., Ibraheem, H. R., Salama, M. A., Sharifuddin, A. M., Ahmed, I. D.: Solving vehicle routing problem by using improved genetic algorithm for optimal solution. Journal of Computational Science 21, 255-262 (2017)

3. Coello, C. A., Santos, F. H., Farrera, F. A.: Diseño óptimo de vigas de concreto reforzado mediante algoritmos genéticos. Universidad Autónoma de Chiapas, México, 1-16 (1995)

4. Chakrabarty, B. K.: Model for optimal design of reinforced concrete beams. Computers and Structures 42(3), 447-451 (1992)

5. Osyczka, A.: Multicriteria optimization for engineering design. En: John S. Gero, editor, Design Optimization, Academic Press, 193-227 (1985)

6. Prakash, A., Agarwala, S. K., Singh, K. K.: Optimum design of reinforced concrete sections. Computers and Structures 30(4), 1009-1011 (1988)

7. Dan, S.: Evolutionary optimization algorithms. Wiley, EE.UU. (2013)

8. Merchán, E. A.: Soft-computing techniques in the trajectory planning of robot manipulators sharing a common workspace. University of Sheffield, U.K., 1-204 (2005)

9. IMCA.: Manual en construcción en acero, Limusa, México (2002)

10. Mott, R. L.: Resistencias de materiales. Pearson, México (2009) 